\title{
Improvement of Spatial Luminance Uniformity in Emitted Light from Flexible Backlight Using Notch-Type Variable Light Distribution Films
}

\author{
Takumi SHOJI $^{\dagger a)}$, Yosei SHIBATA ${ }^{\dagger}$, Nonmembers, Takahiro ISHINABE ${ }^{\dagger}$, Member, $^{\prime}$ \\ and Hideo FUJIKAKE ${ }^{\dagger}$, Fellow
}

SUMMARY In recent years, flexible liquid crystal displays (LCDs) have attracted much attention due to their thin and lightweight designs, as well as their ease of installation compared with conventional flat-panel LCDs. Most LCDs require backlight units (BLUs) to yield high-quality images. However, the luminance uniformity of flexible BLUs is drastically reduced in the curved state, as the light emitted from a typical BLU is mainly directed in the normal direction. Conventional BLUs do not provide the image quality of flexible BLUs due to uneven luminance distribution from the perspective of the observer. To overcome these issues, here we introduce a novel oblique-angled notched film for improved light distribution of a conventional BLU. The notched structure of the proposed film exhibits V-shaped split behavior during curvature. This elastic deformation is expected to compensate for the spatial luminance of the light emitted from the BLU. We investigated the design requirements for the proposed film based on geometrical calculations. The luminance distribution of a flexible BLU was then simulated using the proposed film, based on geometrical optics theory. The simulation results confirmed that the direction of travel of the light is controllable according to the total internal reflection of the proposed film, thus improving the luminance uniformity of the BLU in a convex-curved state. Based on these results, the proposed approach is expected to improve the luminance uniformity of convex-curved flexible LCDs.

key words: backlight, flexible LCD, light distribution, luminance uniformity

\section{Introduction}

\subsection{Flexible Liquid Crystal Displays}

With the continual improvement of material technologies, there has been an increasing demand for high-performance deformable electronic displays. For example, it is now possible to realize flexible wearable displays that can be placed around an arm. The development of new types of liquid crystals for flexible liquid crystal displays (LCDs) [1] is expected to improve the reliability and applicability of such LCD devices.

The basic structure of a flexible LCD is shown in Fig. 1. LC materials have dielectric anisotropy and birefringence characteristics; therefore, it is possible to alter the polarization state of transmitted light via voltage applied to the LC [2]. In LC panels, LC materials are sandwiched

Manuscript received March 15, 2019.

Manuscript revised June 6, 2019.

${ }^{\dagger}$ The authors are with the Department of Electronic Engineering, Tohoku University, Sendai-shi, 980-8579 Japan.

a) E-mail: takumi.shoji.r5@dc.tohoku.ac.jp

DOI: $10.1587 /$ transele.2019DII0004

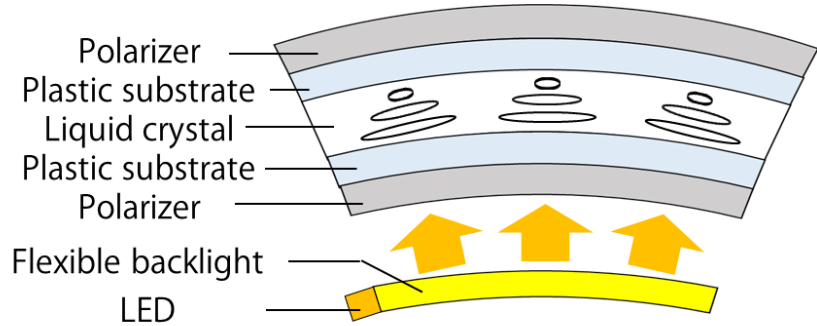

Fig. 1 Schematic illustration of a flexible liquid crystal display (LCD) structure. LED: light-emitting diode.

between two flexible plastic substrates. In addition, two polarizers with crossed-Nicols states act as a light shutter for light emitted from the flexible backlight unit (BLU). A flexible backlight [3] is necessary for transmissive-type LCDs, as LC materials are commonly nonemissive. Therefore, the development of a flexible backlight with uniform luminance is an important challenge for realizing high-quality flexible LCDs.

\subsection{Technical Issues of Flexible Backlight Units}

Flexible BLUs have been investigated extensively and are used in numerous devices; the flexible BLU used in mobile devices, such as cell phones, is the edge-type BLU (Fig. 2) [4], [5]. This BLU emits uniform light to the surface via a light guide plate (LGP). Light from the LGP is directed in a normal direction by two crossed-prism films attached to the diffusing film. [4], [6]. The edge-type BLU has approximately a cone shape solid angle for emergent light [4].

Figure 3 shows the effect of the prism films on the angular luminance distribution; the luminance along the normal direction is especially strong, leading to effective light utilization efficiency when viewed from above. Notably, the optical characteristics of the prism film are maintained even in films in a curved state [6]. However, the most commonly used flexible backlight shows a poor luminance distribution in a curved state, due to the characteristics of the prism film. Compared with backlighting in the flat state, the luminance uniformity in the convex-curved state decreases at both ends of the backlight, as shown in Fig. 4. This is because the main direction of travel of emitted light is at approximately large 
angle with respect to the observer.

To overcome this issue, a novel optical compensation technique is required to maintain luminance uniformity even in a curved state. Several optical compensation methods for the curved state have been reported. For example, a small lens film was mounted on a collimated backlight for discrete illumination of the beam light [7]. The disadvantage of this method is that it requires a precisely controlled alignment process and structural changes in the LGP.

Here, we introduce an oblique-angled notched film for application to the conventional flexible BLU design, for improved light distribution. The alignment process is not needed in the introduced method. The notched structure of the film exhibits a V-shaped split behavior during curvature that compensates for the spatial luminance of the light emitted from the BLU. In this paper, we explain the operating principle of the proposed film and introduce a structural design to achieve the desired luminance distribution, based on optical simulations.

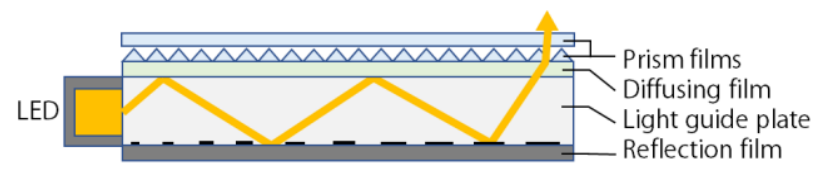

Fig. 2 Schematic illustration of the edge-type flexible backlight unit.

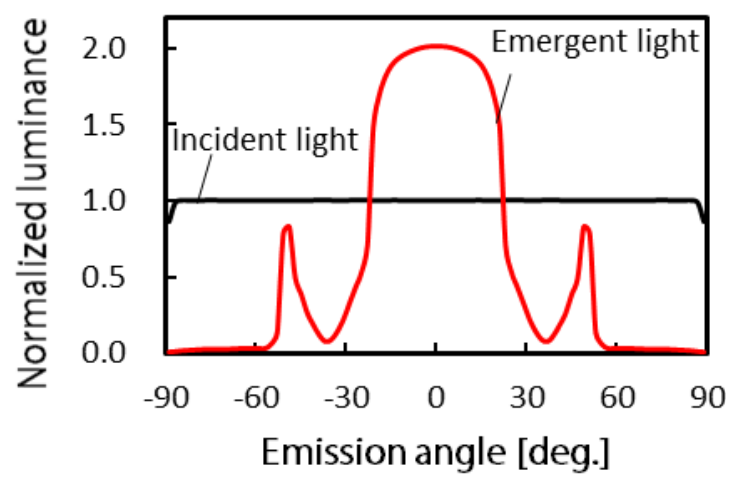

Fig. 3 Effect of prism films on the angular luminance distribution.

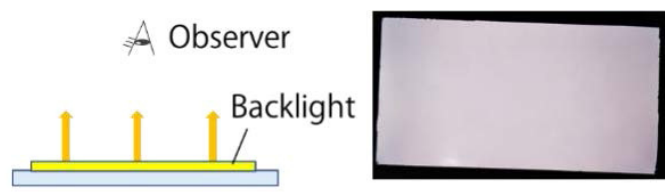

(a) Flat state

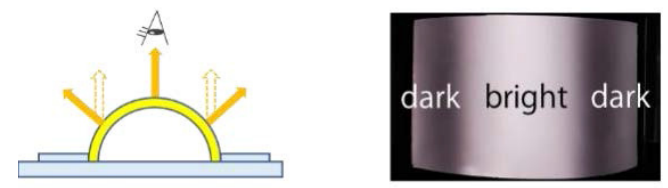

(b) Curved state

Fig. 4 Luminance uniformity of the flexible backlight.

\section{Notch-Type Light Distribution Film}

\subsection{Principle of Light Distribution Control via Notch-Type Light Distribution Film}

The proposed light distribution film is made of thin elastic materials having a high refractive index, such as silicone elastomer. This is important for improving the luminance uniformity of the flexible backlight in the curved state. Here, we considered a notched film structure with an oblique angle relative to the elastomer plate surface, as shown in Fig. 5. When the light emitted from the flat-state backlight enters the film, it continues in a straight line because the notch is closed (Fig. 6(a)). In contrast, the direction of travel of partially transmitted light changes when the light emitted from the curved backlight enters the film (Fig. 6(b)); this occurs because the notches are open. The notches form a wedgeshaped gap due to the convex curvature. Assuming that the

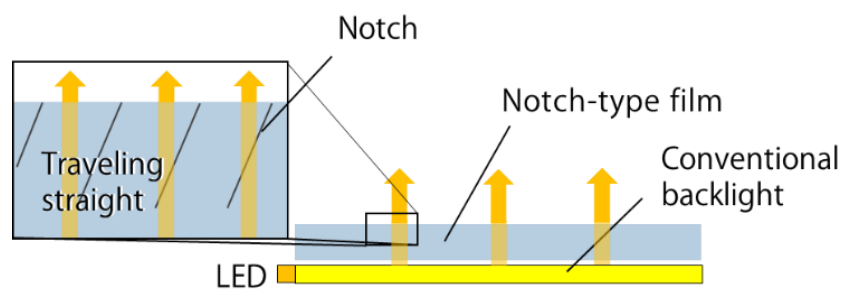

(a) Flat state

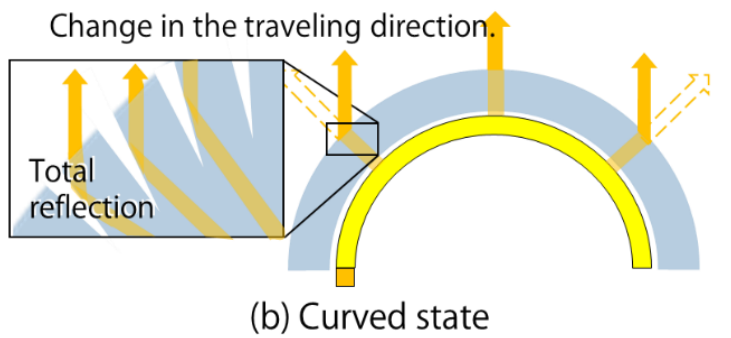

Fig. 5 Principle of collection of light emitted through the notch-type light distribution films.

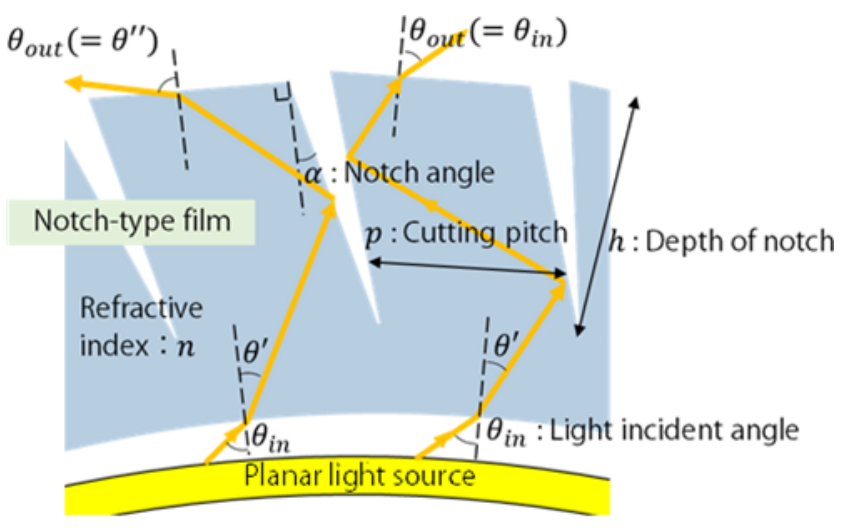

$\begin{array}{ll}\text { (a) Odd number of TIRs } & \text { (b) Even number of TIRs }\end{array}$

Fig. 6 Schematic diagram of the light path in the proposed notch-type film. TIR: total internal reflection. 
compressive deformation can be ignored in the proposed optical film, the parts of the film sandwiched by the notches become parallelograms. Consequently, total internal reflection (TIR) and refraction occur, as described by Snell's law [8]. Thus, the introduction of notched, oblique-angled elastomer film to the BLU directs the emitted light to the observer appropriately for improved luminance.

We investigated several factors in an attempt to further optimize luminance uniformity in the curved state, specifically, the effect of TIR in the film on the direction of the emergent light, the condition for directing the light to the observer, and the light-emitting direction required for the curved backlight.

2.2 Investigation of the Effect of TIR in the Film on the Direction of Light Emission

Figure 6 shows the direction of travel of the incident light through the proposed notch-type film. Here, the normal direction of the light emitting surface is defined as $0^{\circ}$. Assuming that the refractive index of the proposed film is $n$, the incident light at the angle $\theta_{\text {in }}$ travels in the direction of $\theta^{\prime}=\arcsin \left((1 / n) \cdot \sin \theta_{\text {in }}\right)$ via refraction at the interface. If the light traveling in the $\theta^{\prime}$ direction enters the notch (notch angle: $\alpha$ ), its direction of travel changes to $-\theta^{\prime}+2 \alpha$ via TIR. When the light enters the notch again at a tilted angle $\alpha$, its direction of travel returns to the original angle $\theta^{\prime}$. The light emission angle $\left(\theta_{\text {out }}\right)$ is equal to the incident angle $\left(\theta_{\text {in }}\right)$ when the number of TIRs inside the film is even. In the case of an odd number of TIRs, $\theta_{\text {out }}$ is equal to $\theta^{\prime \prime}$, as expressed in Eq. (1):

$$
\theta^{\prime \prime}=\arcsin \left(n \cdot \sin \left(-\theta^{\prime}+2 \alpha\right)\right)
$$

This equation describes the optical design required to direct the light towards $\theta^{\prime \prime}$, in which the incident ray shows an odd number of reflections in the notched film. In addition, realization of the following optical design leads to an improvement in the luminance distribution, as $\theta^{\prime \prime}$ is directed to the observer in the curved state when the number of TIRs is odd.

\subsection{Design of the Proposed Film to Direct Light to the Ob- server}

On the basis of the description provided in the previous section, we investigated the optical conditions required for an odd number of TIRs. According to Fig. 6, there are three main parameters related to the notch structure of the film: the notch angle $\alpha$, the cutting pitch $p$, and the notch depth $h$. The incident angle of light from the backlight $\left(\theta_{i n}\right)$ is also related to the number of TIRs. The distance traveled by the light in the film thickness direction, for a single TIR, is $\frac{p}{\tan \left(\theta^{\prime}+2 \alpha\right)-\tan \alpha}$ after an even number of TIRs and $\frac{p}{\tan \theta^{\prime}+\tan \alpha}$ after an odd number of TIRs. Therefore, the average distance traveled in the film thickness direction for a single TIR $d_{\text {average }}$ is expressed by the following equation:

$$
d_{\text {average }}=\frac{p}{2}\left(\frac{1}{\tan \left(\theta^{\prime}+2 \alpha\right)-\tan \alpha}+\frac{1}{\tan \theta^{\prime}+\tan \alpha}\right)
$$

The condition for maximizing the amount of emergent light in the $\theta^{\prime \prime}$ direction is that the depth of notch $\mathrm{h}$ is equal to the average distance traveled by the light in the film thickness direction during an odd number of TIRs. Therefore, the most desirable condition is given by:

$$
\begin{aligned}
& h=k \cdot d_{\text {average }} \\
& \frac{h}{p}=\frac{k}{2}\left(\frac{1}{\tan \left(\theta^{\prime}+2 \alpha\right)-\tan \alpha}+\frac{1}{\tan \theta^{\prime}+\tan \alpha}\right)
\end{aligned}
$$

where $k$ is the number of TIRs in the film, such that $k$ is an odd number. Optimization of the ratio between $h$ and $p$ is also needed to fulfill Eq. (4), because the notch angle $\alpha$ is uniquely determined based on the light-emitting direction, as given in Eq. (1). This means that the film thickness can be reduced by decreasing the cutting pitch $p$. Furthermore, the number of TIRs, $k$, is inversely proportional to the cutting pitch $p$. During fabrication of the proposed film, a large cutting pitch is desirable due to the simplicity of the process. Therefore, we took $k$ as 1 and attempted to control the lightemitting direction.

\subsection{Geometric Design of the Notch Angle}

To direct the light to the observer, it is necessary to fully understand the observer's position. For a convex-curved flexible backlight surface, angle $\varphi$ (Fig. 7) depends on three parameters: the observation distance $(d)$, the curvature radius $(r)$, and the spatial position of the backlight $(x)$. Then, $\varphi$ can be expressed by

$$
\varphi=\frac{x}{r}+\arctan \left(\frac{\sin \frac{x}{r}}{\frac{d}{r}+1-\cos \frac{x}{r}}\right)
$$

To optimize luminance uniformity in the curved state, angle $\varphi$ must be consistent with $\theta^{\prime \prime}$. Therefore, the notch angle $\alpha$ must fulfill the following equation:

$$
\alpha=\frac{1}{2}\left(\theta^{\prime}+\arcsin \left(\frac{1}{n} \sin \left(\frac{x}{r}+\arctan \left(\frac{\sin \frac{x}{r}}{\frac{d}{r}+1-\cos \frac{x}{r}}\right)\right)\right)\right)
$$

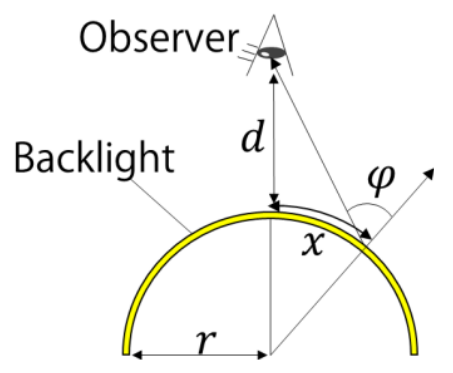

Fig. 7 Observation angle of a convex-curved surface. 
The notch angle $\alpha$ must also change gradually within the backlight plane, as the observation angle $\varphi$ depends on the spatial position $x$.

\section{Optical Simulation}

\subsection{Investigation of the Angle of Light Emitted from the Proposed Film}

Here we discuss the distribution of emitted light for the designed notch-type light distribution film, mounted on a conventional flexible backlight with prism films. As an example, we consider that light is emitted in the direction of $\theta^{\prime \prime}=30^{\circ}$. The film material is silicone elastomer, with a refractive index of $n=1.41$. This value is obtained by optical experiments using light wavelength of $550 \mathrm{~nm}$. We achieve maximum luminance on surface normal direction in conventional planar BLU. Therefore, if the incident light angle $\theta_{\text {in }}$ is represented by $0^{\circ}$, the most suitable notch angle calculated from Eq. (1) is $10.4^{\circ}$. On the basis of this value, Eq. (4) is fulfilled using a notch depth of $h=500$ $\mu m$ and a cutting pitch of $p=94.7 \mu m$. To estimate the light distribution characteristics of the emitted light in the curved state, we used ray-tracing simulations [9] performed with LightTools software (Synopsys Corp., Mountain View, CA, USA). The backlight model for the ray-tracing calculation included a sheet-like light source, uniform luminance and the two crossed-prism films. The light source has Lambertian profile.

Figure 8 shows the simulation results; black and red lines shows the angular characteristics of the conventional backlight without and with the proposed film, respectively. The notched film directs some proportion of the emitted light toward the observer at $30^{\circ}$. As shown in the figure, luminance in the $30^{\circ}$ direction increased by 5.9-fold compared to that of without film. This luminance corresponds to $81 \%$ of that emitted along the normal direction.

We clarified that mounting the proposed film on a conventional backlight can control the angle of the emitted light in the curved state.

\subsection{Luminance Uniformity of a Convex-Curved BLU with} a Notch-Type Film

The effect of the proposed notch-type film on luminance was verified for transmissive flexible LCDs in a curved state. To determine the effect, we evaluated the uniformity of the spatial luminance of the curved backlight with the film using ray-tracing analysis.

Prior to the simulation, the required size of the backlight, i.e., $59 \times 104 \mathrm{~mm}$, was determined for a wearable display that could be used around arm. This size corresponds to that of commonly used smartphones. We assumed the following conditions for the emitted light: curvature radius of $r=26 \mathrm{~mm}$ and observation distance of $d=30 \mathrm{~cm}$. In addition, the thickness and depth of the notch-type film were $1.1 \mathrm{~mm}$ and $500 \mu \mathrm{m}$, respectively. Under these conditions, the notch angle $\alpha$ and cutting pitch $p$ were determined by Eqs. (6) and (4), respectively. The incident light on the film is represented by $\theta=0^{\circ}$. To simplify the simulation model, the notch angle $\alpha$ and cutting pitch $p$ are the approximate values shown in Figs. 9 and 10. The simulation model is as shown in Fig. 11, with the planar light source showed Lambertian light distribution.

Figure 12 shows the simulation results of the spatial luminance across the center line of the BLU. The spatial luminance of the incident light to the notch film was nonuniform. With the notch film, the luminance uniformity of

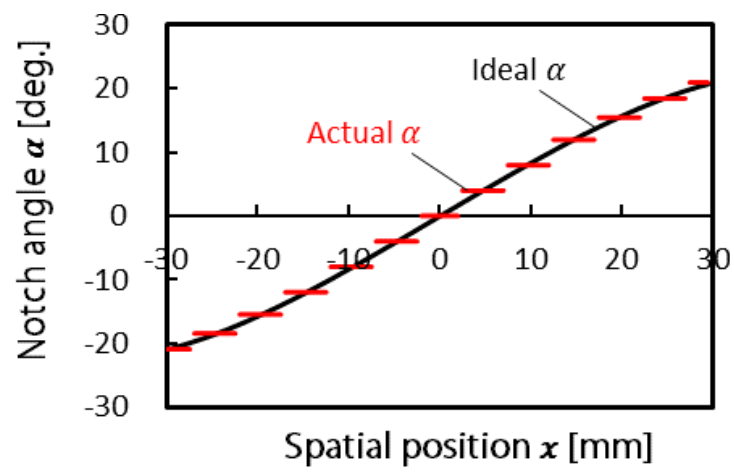

Fig. 9 Approximation of notch angle $\alpha$.

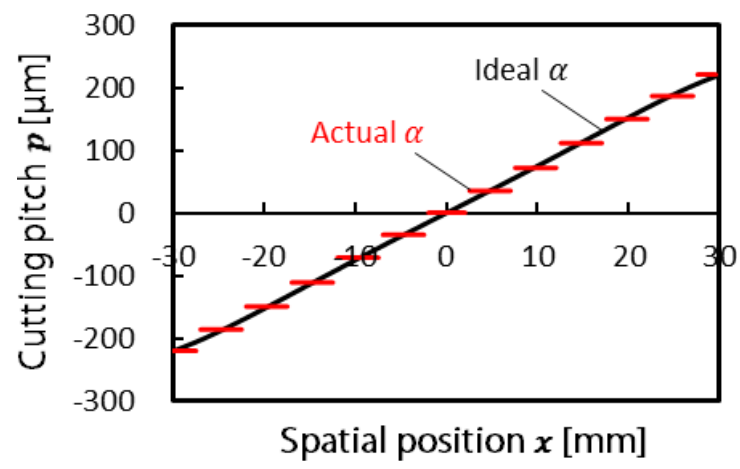

Fig. 10 Approximation of cutting pitch $p$.
Fig. 8 Angular luminance distribution aiming for ight emission in the

$30^{\circ}$ direction.

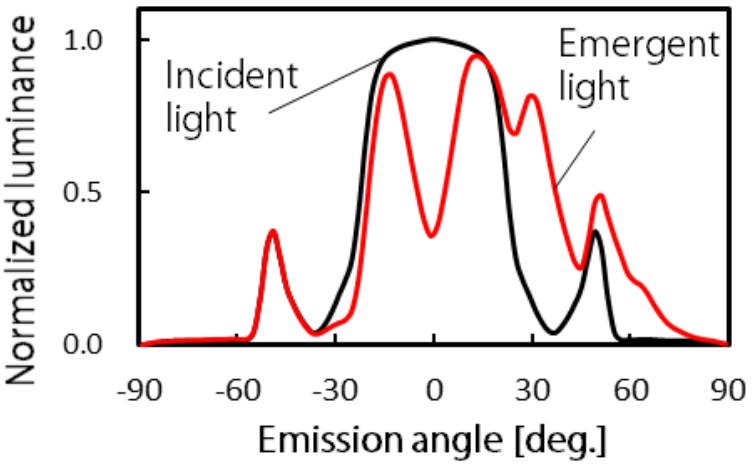




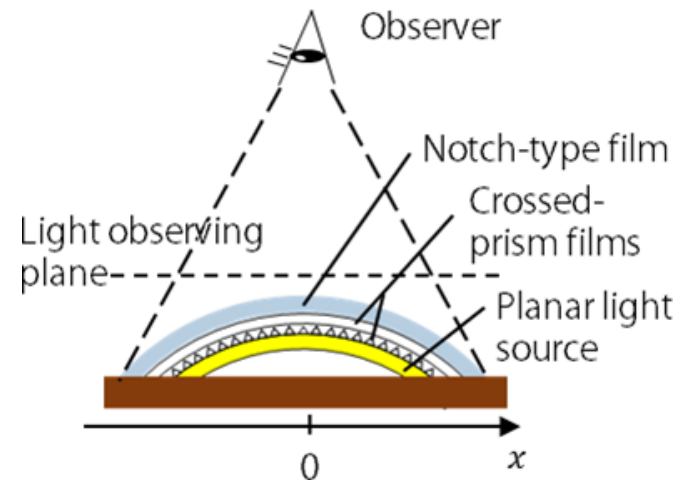

Fig. 11 Ray-tracing simulation model.

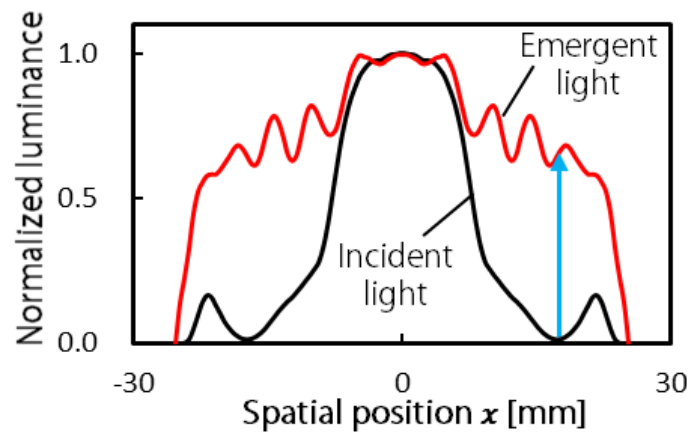

Fig. 12 Simulation results of spatial luminance across the center line of the backlight unit (BLU) perpendicular to the curved axis.
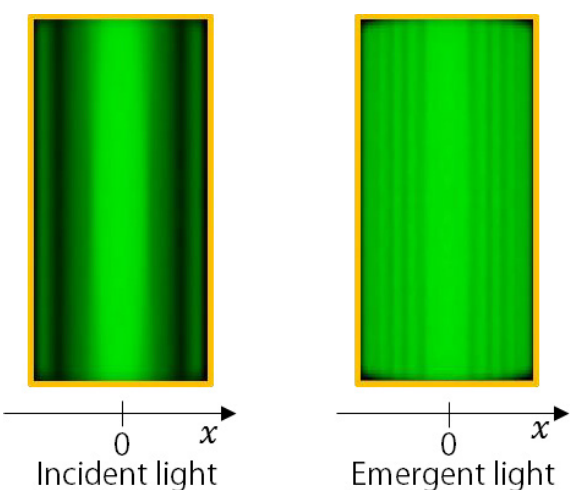

Fig. 13 Simulation results of spatial luminance across the center line of the BLU perpendicular to the curved axis.

the emergent luminance was enhanced, because the luminance reduction at the ends is improved. The increase in luminance across the surface is due to the notch film delivering incident light that is originally directed to an angle not visible to the observer. We assumed that the slight unevenness in the distribution of the luminance was caused by discrete modeling of the notch angle $\alpha$ and cutting pitch $p$. Figure 13 also shows the simulation results for spatial luminance over the entire observation surface, represented as 256 tones. These results correspond to the appearance when the curved backlight is observed from a vertical (overhead) position, as shown in Fig. 4. Visual inspection confirmed the improvement in luminance uniformity with use of the notch-type film (Fig. 13). From the above results, we can conclude that the spatial luminance uniformity of curved backlight LCDs can be improved by incorporating notchtype film into conventional backlights.

\section{Conclusion}

The purpose of our research was to improve the luminance uniformity of flexible BLUs in the convex curved state. Here a novel method was employed to apply a notch-type film to a conventional BLU for resolving the uniformity issue. The requirements for the notch film were determined based on geometrical calculations and simulations. Improvement in the luminance uniformity was achieved by incorporating the proposed film into the curved BLU in the optical simulations. It is expected that this approach improves the luminance uniformity of convex-curved flexible LCDs. The next phase of our research will involve further optimization of the proposed design, with a focus on the viewing angle, as well as evaluation of the steps necessary for device fabrication.

\section{References}

[1] H. Fujikake, H. Sato, T. Murashige, Y. Fujisaki, H. Kikuchi, and T. Kirita, "Flexible field-sequential-color FLC displays using a bendable back light sheet with LCD chips," Proc. IDW, LCT5-1, pp.871-874, 2005.

[2] H. Takezoe and K. Miyachi, "Essential in Chemistry 19 Liquid Crystals: From Fundamentals to Frontiers of Science and Display Technologies," Kyoritsu Shuppan, 2017.

[3] H. Fujikake, "Advanced flexible liquid-crystal display technologies," SPIE Newsroom, 10.1117/2.1200811.1376, page1/3-3/3, 2008.11.

[4] K. Käläntär, "LCD Backlighting Technologies," CMC publishing, 2006.

[5] K. Käläntär, "LED Backlighting Technologies," CMC publishing, 2010.

[6] B. Xie, R. Hu, Q. Chen, X, Yu, D. Wu, K. Wang, and X. Luo, "Design of a brightness-enhancement-film-adaptive freeform lens to enhance overall performance in direct-lit light-emitting diode backlighting," Proc. Optical Society of America, vol.54, no.17, pp.5542-5548, June 102015.

[7] D.J. Montgomery, H. Walton, T. Ishida, and Y. Tsuda, "A variable curve backlight," Proc. SID, 4.1, pp.12-15, 2009.

[8] Y. Bao, N. Hamaguchi, and T. Gotoh, "Stereoscopic display using total reflection," Proc. The Journal ITE, vol.53, no.8, pp.1153-1158, 1999.

[9] M. Ota, A. Takeuchi, and T. Oguchi, "Applied Graphics," ASCII Publishing, p.41, 1993. 


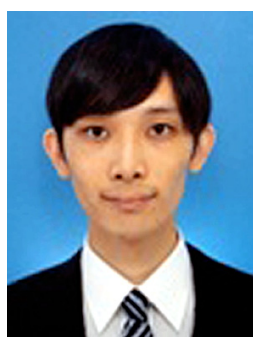

Takumi Shoji received the B.E. degree from Tohoku University, Sendai, Japan, in 2018. Now, he is a graduate student in the Department of Electronic Engineering, Graduate School of Engineering, Tohoku University. He is engaged in the optical design of backlight for LCDs. He received the Outstanding Poster Paper Award from IDW in 2018.

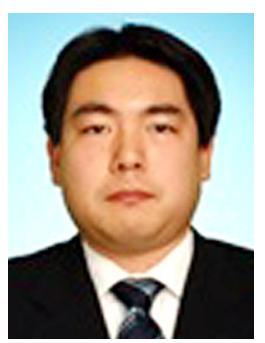

Yosei Shibata received his $\mathrm{PhD}$ degree in engineering from Tokyo Institute of Technology (Japan) in March 2013. He has research interests on organic semiconductor devices such as organic transistors and organic photovoltaics. Then he joined the National Institute of Advanced Industrial Science and Technologies (AIST, Japan) as a postdoctoral position. In October 2015, he joined the Department of Electronics of Tohoku University as an Assistant Professor.

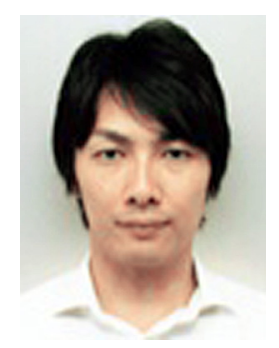

Takahiro Ishinabe received his B.S., M.S., and $\mathrm{Ph}$. D. degrees in Electronic Engineering from Tohoku University, Sendai, Japan, in 1995, 1997 and 2000, respectively. From 2000 to 2002, he was a Research Fellow of the Japan Society for the Promotion of Science and from 2003 to 2012, he was an Assistant Professor, and since 2013, he has been an Associate Professor in the Department of Electronics, Graduate school of Engineering, Tohoku University. He has also been a Visiting Professor in the CREOL, The College of Optics and Photonics, University of Central Florida from 2010 to 2011. He has been performing a research on advanced liquid crystal displays such as wide viewing angle $L C D$, reflective full-color LCD, field sequential color LCD and flexible LCD.

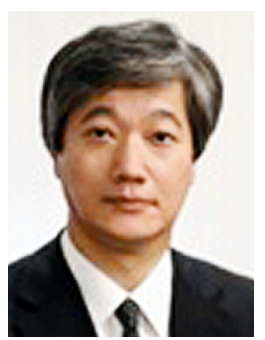

Hideo Fujikake received M.E and Ph.D. degrees from Tohoku University, Japan, in 1985 and 2003, respectively. In 1985, he joined Japan Broadcasting Corporation (NHK). In 1988-2012, he was with NHK Science and Technology Research Laboratories. Since 2012, he has been a professor at Department of Electronic Engineering, Tohoku University. He received Best Paper Award from Institute of Electronics, Information and Communication Engineers (IEICE) in 2001, Best Paper Award from Japanese Liquid Crystal Society (JLCS) in 2001 and 2015, NiwaTakayanagi Best Paper Awards from Institute of Image Information and Television Engineers of Japan (ITE) in 2003 and 2009, and Electronics Society Award from IEICE in 2013. His current interest is concerned with flexible liquid crystal displays. He also served as a General Vice Chair in International Display Workshops in 2015-2016, a Japan Chapter Chair in IEEE Consumer Electronics Society in 2012-2014, and a Vice President of Japanese Liquid Crystal Society in 2015-2016. He is an IEICE fellow since 2015, and ITE fellow since 2016. 